\title{
DeepFEC: Energy Consumption Prediction under Real-World Driving Conditions for Smart Cities
}

\author{
Sayda Elmi \\ National University of Singapore \\ School of Computing \\ Singapore \\ saida@comp.nus.edu.sg
}

\author{
Kian-Lee Tan \\ National University of Singapore \\ School of Computing \\ Singapore \\ tankl@comp.nus.edu.sg
}

\begin{abstract}
The status of air pollution is serious all over the world. Analysing and predicting vehicle energy consumption becomes a major concern. Vehicle energy consumption depends not only on speed but also on a number of external factors such as road topology, traffic, driving style, etc. Obtaining the cost for each link (i.e., link energy consumption) in road networks plays a key role in energy-optimal route planning process. This paper presents a novel framework that identifies vehicle/driving environment-dependent factors to predict energy consumption over a road network based on historical consumption data for different vehicle types. We design a deep-learning-based structure, called DeepFEC, to forecast accurate energy consumption in each and every road in a city based on real traffic conditions. A residual neural network and recurrent neural network are employed to model the spatial and temporal closeness, respectively. Static vehicle data reflecting vehicle type, vehicle weight, engine configuration and displacement are also learned. The outputs of these neural networks are dynamically aggregated to improve the spatially correlated time series data forecasting. Extensive experiments conducted on a diverse fleet consisting of 264 gasoline vehicles, 92 Hybrid Electric Vehicles, and 27 Plug-in Hybrid Electric Vehicles/Electric Vehicles drove in Michigan road network, show that our proposed deep learning algorithm significantly outperforms the state-of-the-art prediction algorithms. To make the results reproductible, the code, the used data and details of the experimental setup are made available online at https://github.com/ElmiSay/DeepFEC.
\end{abstract}

\section{CCS CONCEPTS}

- Computer systems organization $\rightarrow$ Embedded systems; Redundancy; datasets; • Neural Networks $\rightarrow$ Network reliability.

\section{KEYWORDS}

Smart City, Energy Consumption, Prediction, Road Network, Traffic Conditions, Spatio-temporal Features.

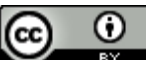

This work is licensed under a Creative Commons Attribution International 4.0 License.

WWW'21, April 19-23, 2021, Ljubljana, Slovenia

(C) 2021 IW3C2 (International World Wide Web Conference Committee), published under Creative Commons CC-BY 4.0 License.

ACM ISBN 978-1-4503-8312-7/21/04.

https://doi.org/10.1145/3442381.3449983
}

\section{ACM Reference Format:}

Sayda Elmi and Kian-Lee Tan. 2021. DeepFEC: Energy Consumption Prediction under Real-World Driving Conditions for Smart Cities. In Proceedings of the Web Conference 2021 (WWW'21), April 19-23, 2021, Ljubljana, Slovenia. ACM, New York, NY, USA, 11 pages. https://doi.org/10.1145/3442381.3449983

\section{INTRODUCTION}

Increasing attention has been paid to analyse and predict the energy consumption for many reasons: (i) To cope with the problem of pollutant emissions caused by gasoline and diesel powered vehicles since it has been estimated that $90 \%$ of urban air pollution in fast-growing cities is attributed to vehicle emissions [34]. (ii) The price of petrol has always been climbing upwards and the total economic losses due to traffic congestion in 439 US cities in 2017 was 3.3 billion gallons of fuel waste [20]. Therefore, it is apparent that effective techniques for estimating energy consumption are essential in in order to reduce unnecessary fuel wastage. (iii) Drivers of Electric Vehicles (EV) must make plans based on estimated energy consumption because they fear that an EV might run out of power and stop on the road.

Some intelligent transportation systems (ITS) solutions such as energy-efficient route planning and charging facilities guidance systems for EV have been proposed to help drivers to optimize travel route and find charging station timely, respectively. Prediction of energy consumption is a prerequisite in route planning and navigation systems [14]. In addition, predicting energy consumption rate is a useful tool to facilitate dynamic adjustment of toll fees so that directions towards high consumption rate links would pay a higher toll fees. Therefore, it is of great importance to accurately predict energy consumption not only for electric vehicles but also other vehicle types such as: Internal Combustion Engines Vehicles (ICE), Hybrid Electric Vehicles (HEV), and Plug-in Hybrid Electric Vehicles (PHEV).

Although all electronic maps and online car-hailing services provide the travel time estimation such as Google Map, Uber and Didi, they do not provide the estimation of energy consumption. Energy Consumption Prediction (ECP) is a crucial task to get accurate energy consumption estimation. Therefore, the problem of ECP has attracted many researcher's attention and been widely studied in intelligent transportation systems but providing an accurate energy prediction is still very challenging because it is highly related to many factors. Furthermore, in the literature, each vehicle type is modeled separately which is not convenient for transportation companies in their real-life applications. To the best of our knowledge, our model is the first that predicts fuel/energy consumption of different vehicle types. This can be very useful for the 
transportation network companies such as taxi or bus markets that can hold different taxi/bus vehicle types (EV, ICE, etc) and want to predict the consumption for all the vehicles circulating on the road network. With our generalised architecture, no need to use a separate model for each vehicle type. In addition, we provide the energy consumption rate per link which is very useful to help drivers optimize travel routes and find reserving or charging stations timely.

In real-world, vehicle energy consumption is variable and dependent on a number of external factors such as road topology, traffic, vehicle speed, driving style, ambient temperature, road conditions, road grade, etc.

In this paper, we aim to predict the energy consumption in the near future related to each road segment in a large road network based on historical observations. For this end, we propose a deep spatio-temporal and contextual neural network called DeepFEC, to provide accurate energy consumption prediction based on capturing the following patterns:

Contextual Patterns: The contextual factors, mainly represented by static vehicle data such as vehicle performance, vehicle type, vehicle weight, engine configuration and displacement, may effect the prediction of energy consumption.

Spatial Patterns: Different road segments have strong dependency with each other. For example, a congestion in a given road will affect surrounding areas especially adjacent roads. Since energy consumption is highly related to traffic congestion, it will be affected on a road segment if congestion occurs in its surrounding area. Therefore, the energy consumption prediction model must deal with spatial correlations among different road segments.

Temporal Patterns: The temporal information is also a key factor that has a great impact on traffic condition. For example, the traffic condition will be much more worse in the rush hours than in the off-peak hours as well as in the weekend. Thus, the energy consumption is highly correlated with the time period in a year, a month and a day, rush hour or holiday.

The goal of this paper is to detect correlations between the the road network, the traffic condition, the vehicle parameters, and its energy consumption.

\section{RELATED WORK}

Over the past decades, number of intelligent traffic prediction systems have been proposed. Some are dedicated to travel time estimation $[15,26,30]$ and others are for traffic speed prediction [16, 17]. Since the problem of energy is becoming more prominent, energy consumption studies are nowadays of great importance in traffic systems. Emissions modeling [2, 4] have been improved to allow better evaluations of transportation operational effects. In particular, instantaneous or modal emissions models [24, 25] have been developed for a variety of vehicles. Then, there have been several major categories of methods used to resolve ECP. One is to focus on the characteristics of the engine [22].Energy consumption can be calculated based on engine data combined with the corresponding engine cycle conditions and vehicle technical parameters [23, 33]. The second is to use the carbon balance method to calculate the energy consumption. The third one is building an energy consumption prediction model using independent variables affecting the energy consumption, based on real vehicle observations [12,21]. To analyse vehicle performance on large amount of data, Perera and Mo [19] developed a big data framework for managing ship data to conduct pre-processing and post-processing. Data pre-processing includes sensor error detection, data classification, and data compression, whereas data post-processing includes data expansion, integration verification, and data regeneration. However, they derive a mathematical model of the relationship among the parameters using a simple regression model, and no study has been conducted to verify the accuracy of energy consumption prediction.

As demonstrated in several studies [1, 4], relationship between vehicle fuel consumption/emissions and other factors including vehicle characteristics (e.g., vehicle type and model year), roadway characteristics (e.g., roadway type and vertical grade), and traffic conditions (e.g., speed and congestion level), is not linear, i.e., the relationship between the predictors and responses becomes increasingly complex and includes high dimensional data, interactions, linear and non-linear relationships. This is important to be noted while creating predictive models since physical and statistics models are not able to capture the complex non-linearity between variables. More recently, machine learning has been shown to outperform classical statistical models and are suitable for fuel consumption prediction, as the model can be developed by learning the patterns in available data. However, traditionally, ML techniques have not been applied to this domain since they require extensive data. Fortunately, this is no longer an issue today as with the advancement of Global Positioning System (GPS) based tracking devices, precision fuel sensors, vehicle owners are now able to capture high resolution, multi-variate time series data-sets related to a vehicle location, speed, engine conditions, and fuel consumption. Extensive input data are now available and easily collected which can be used to very accurately estimate energy/emissions from vehicles and be suitable for real-time applications as in roadway navigation.

Artificial Neural Networks (ANNs) have been actively studied in transportation field to improve the accuracy of regression models [29], including high- dimensional factors and complex interactive relationships between factors. They are a structured technique for modeling highly nonlinear and dynamic systems and simple linear systems. ANNs are helpful in developing an appropriate model when the physical relationships are complex or when the nature of the event has unknown properties. Several researchers have applied regression analysis using ANNs in energy consumption prediction. Yiman et al. [7] explored the energy consumption pattern and and congestion pattern based on large samples of historical floating vehicle data by using ANNs. However, the power of deep learning methods has not yet been exploited in energy consumption prediction in terms of the depth of the model architecture. Compared to existing ECP models, our hybrid architecture fully investigates the potential of deep learning techniques in terms of depth by taking advantages of both residual neural network (ResNet) and recurrent neural network (RNN) which are exploited to learn spatial and temporal patterns, respectively.

RNN have been successfully applied to solve traffic problems $[9,26]$ since it can automatically reserve historical sequence information in its model structure. Authors in [30] introduced a bidirectional LSTM (Bi-LSTM) based approach by utilizing backward information to improve the memory capability. These methods 
achieved better results than statistical methods. However, these attempts still mainly focus on the temporal correlations of traffic evolution at a single location. Therefore, convolutional neural networks $(\mathrm{CNN})$ have been successfully applied in traffic problems $[17,32]$ considering the spatial correlations derived from the road network. To have a very deep structure modeling the spatial features, authors in $[15,31]$, proposed the residual learning (ResNet).

To provide more accurate prediction, our proposed model DeepFEC combines residual neural networks and Bi-LSTM in a rational way to make use of their advantages and then learn contextual dependency from vehicle static data.

\section{PROBLEM DEFINITION}

In this section, we present the problem definition of our paper. For reference, a summary of notations is given in Table 1 .

Table 1: The summary of notations.

\begin{tabular}{cc}
\hline Notation & Definition \\
\hline $\mathcal{R}$ & Road Network, $\mathcal{R}=\left\{r_{1}, r_{2}, \ldots, r_{R}\right\}$ \\
\hline$R$ & The number of road segments or links. \\
\hline$X_{t}$ & EC Vector over time, at one location. \\
\hline$X_{r, t}$ & EC Tensor (over time and road network). \\
\hline$N$ & The number of time-slots. \\
\hline$Y$ & Vehicle Tensor \\
\hline$Z$ & Speed Tensor \\
\hline
\end{tabular}

We assume that cars are equipped with sensors and devices that can capture GPS trajectories along with their time-series data of energy consumption, vehicle speed, and auxiliary power usage.

Definition 3.1. Road Network. We represent a city by a set of road segments $\mathcal{R}=\left\{r_{1}, r_{2}, \ldots, r_{R}\right\}$ based on the latitude and longitude of nodes of intersections, where $R$ denotes the total number of road segments $|\mathcal{R}|$.

At one location, the energy consumption can be represented by the vector $X_{t}$ which is a sequence of energy consumption $x$ with $N$ historical time slots where

$$
X_{t}=\left[x_{t-N}, x_{t-(N-1)}, \ldots, x_{t-2}, x_{t-1}, x_{t}\right]
$$

The traffic condition at one location can be impacted by nearby and faraway locations. Because the energy consumption is highly affected by the traffic condition, we design a spatio-temporal matrix recording consumption data for each road segment in a road network over time.

Definition 3.2. Energy Consumption Tensor. Given a road network $\mathcal{R}$ and time slots $\left\{t_{1}, t_{2}, \ldots, t_{N}\right\}$, we use a 3 -dimensional tensor $X \in \mathbb{R}^{R \times N \times C}$ where $C$ denotes the dimension of consumption data $x$ for road segments in all the time slots. A combination of $(r, t, x)$ is unique. The matrix can be expressed as follows:

$$
X_{r, t}=\left[\begin{array}{cccc}
x_{r_{1}, t-N} & \ldots & x_{r_{1}, t-1} & x_{r_{1}, t} \\
x_{r_{2}, t-N} & \ldots & x_{r_{2}, t-1} & x_{r_{2}, t} \\
\vdots & \ddots & \vdots & \vdots \\
x_{r_{R-1}, t-N} & \ldots & x_{r_{R-1}, t-1} & x_{r_{R-1}, t} \\
x_{r_{R}, t-N} & \ldots & x_{r_{R}, t-1} & x_{r_{R}, t}
\end{array}\right]
$$

As in equation 2, the row vector contains consumption data in the same road segment from continuous time intervals (from $t$ to $t-N)$, the columns contain consumption data in all $R$ road segments at the same time slot.

Definition 3.3. Vehicle Tensor. We use a 3-dimensional tensor $Y \in \mathbb{R}^{R, N, V}$ to denote the features of vehicles which record energy consumption for $R$ road segments in $N$ time slots. $V$ denotes the dimension of the vehicle feature vector, i.e., vehicle type, vehicle weight, engine configuration and engine displacement. $y_{r, t}$ denotes the feature vector of road segment $r$ in time slot $t$. A combination of $(r, t, y)$ is unique.

Each energy consumption data in $X$ is given by a vehicle recorded in $Y$. Each vehicle has special characteristics that should be considered in capturing the patterns of energy consumption. Detailed information is given in Section 4.

Definition 3.4. Speed Tensor. We use a 3-dimensional tensor $Z \in \mathbb{R}^{R, N, S}$ to denote the speed feature of all road segments in all time slots. $S$ denotes the dimension of the speed feature vector of $R$ road segments in $N$ time slots. The matrix $Z_{r, t}$ represents average speed data recorded for road segments over time.

Problem Definition: Given the feature tensors $X, Y$ and $Z$, presenting consumption, vehicle and speed features, respectively, of the previous $N$ time slots on $R$ road segments, our goal is to predict the energy consumption for all the regions in a city, in the next time slot $t+p$, where $p$ is the number of time slots to be predicted.

Having energy consumption per road helps to make decision about choosing the trip trajectory based not only on distance or travel time but also on energy consumption which is more and more significant since among energy economy improvements, we can list a few: saving money, reducing climate change, reduces oil dependence costs, increasing energy sustainability, etc..

\section{METHODOLOGY}

\subsection{Framework}

As shown in Figure 1, the architecture of DeepFEC comprises four major components which are: (i) a context extraction layer modeling the static vehicle data such as vehicle type, vehicle weight, engine configuration and engine displacement and (ii) several residual layers to model the spatial evolution, (iii) a recurrent layer and more specifically, we use the Bi-LSTM component to model the temporal dependency and (iv) a temperature and periodicity extraction layers to capture weather and periodicity features of the energy consumption, respectively.

To learn from static vehicle data, we use embedding layers to capture patterns from vehicle type (e.g., ICE, HEV, PHEV, EV), vehicle weight, engine configuration and engine displacement. The embedding layer is most commonly used for pure selection, in the case of pre-trained word embedding. But, it has much more to offer, especially dealing with categorical data in neural networks. In our case, it is used as a dense layer to capture correlation between energy consumption and vehicle characteristics.

For learning spatial patterns, DeepFEC employs Residual units since it has very deep structure that can effectively capture the spatial traffic dynamics from nearby and distant regions and its 
impact on energy consumption. As an input, residual layers take the energy consumption data matrix $X_{r, t}$. We also feed the speed matrix $Z_{r, t}$ into the model to help learning features from the strong correlation between traffic speed and energy consumption. The spatial learning component shares the same road network topology with a convolutional neural network in each residual unit. Such structure captures the spatial traffic dynamics of the surrounding areas. After extracting the spatial patterns, we reshape these features to be suitable for time-series learning and feed into RNN layer and more specifically, a Bi-LSTM as a deeper variant of RNN for sequence modeling.

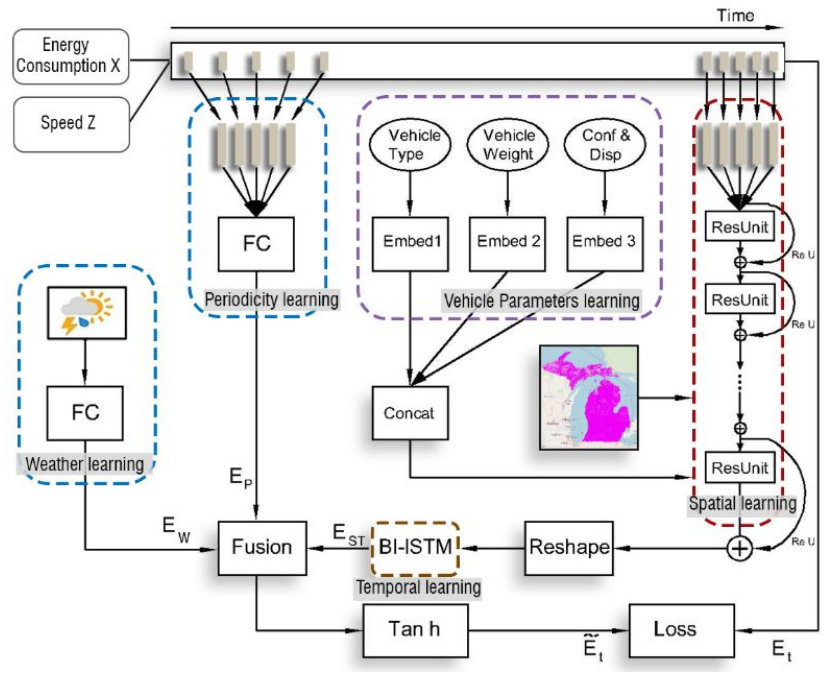

Figure 1: DeepFEC: A Hybrid Architecture.

Energy consumption is significantly impacted by traffic congestion. As a traffic congestion occurring at 8am on one day will be almost the same as the next day at 8am, it means that energy consumption during morning rush hours may be similar on consecutive workdays/weeks, repeating every 24 hours. Hence, we feed the energy consumption and speed vectors of periodic time intervals into fully-connected layers (FC) to capture such temporal dynamics. By a Tanh function, the outputs are aggregated.

\subsection{Vehicle Features Extraction}

In our model, we use one hot encoding method to convert categorical data describing vehicle type, vehicle weight, engine configuration and engine displacement, to a low-dimensional integer vector. These categorical variables are known to hide and mask lots of interesting patterns for the energy consumption prediction and might be the most important features in the model.

One possible way to include the static vehicle data (vehicle type, Engine Configuration, Displacement and Weight) is to use a dense layer. However, each combination of road and time has one vehicle, its speed and its energy emission. Embedding layer efficiently uses lookup operation to match the energy data with static data. We can then let the embedding layer do the job of a dense layer. The embedding layer learns to represent vehicles with a dense vector. It looks up from the learned matrix. Entity Embedding [5] (as shown in Figure 1) is used to deal with categorical variables since it was found that neural networks with entity embedding generate better results and can handle such variables effectively and efficiently. It is most commonly used for pure selection, in the case of pre-trained word embedding. But embedding layer has much more to offer, especially dealing with categorical data in neural network. They play the role of dense layer in such cases, only without activation and bias and is purely dot product. They lookup from learned matrix and output dense vector. Embedding layer learns by updating the matrix (weights). It learns to represent static data vehicles with a dense vector.

\subsection{Spatial and Speed Patterns Extraction}

Thanks to its good performance on capturing spatial features from adjacent pixels, convolution neural network has been widely used in image data analysis [13]. Since energy consumption is highly correlated to traffic and traffic evolution is restricted by the road network (i.e., the traffic condition in a given road is impacted by traffic in adjacent road segments), we thus design a convolution layer that embed the topology of road network into convolution to capture more meaningful spatial features. To have a super deep convolution structure of 100 layers, even over-1000 layers, we use the deep residual learning [11] which has shown superior results on multiple challenging recognition tasks, including image classification, localization, segmentation and object detection [11].

The Road Network as Graph:

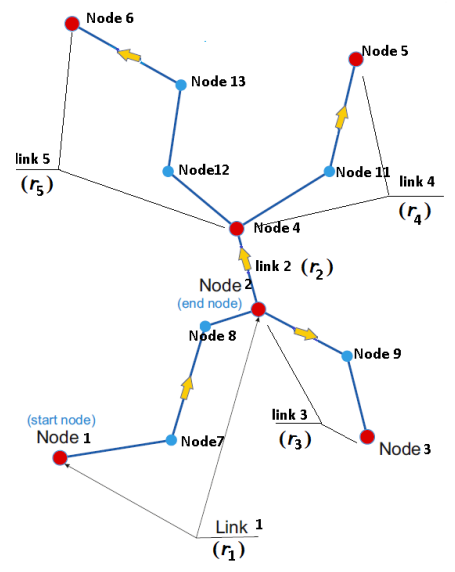

(a) Road Network

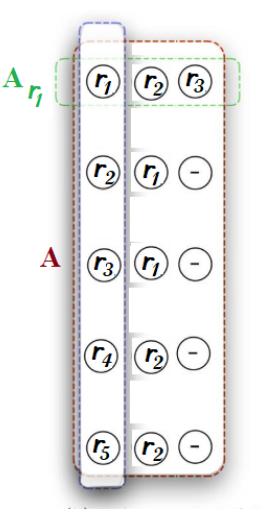

(b) Adjacent Road Matrix
Figure 2: Road Network and Adjacent Road Matrix.

Let $\mathcal{R}$ be the set of road segments $r$. We use r.s and r.e to represent the start and end of the road segment $r \in \mathcal{R}$. As shown in Figure 2a, the road network is a set of road segments, denoted by links in the figure. A link is a pair of nodes between two intersections, e.g., node 1 and node 2 are the start node and the end node, respectively, of link 1 . To mathematically represent the road network topology, we use an adjacent road matrix as shown in Figure $2 \mathrm{~b}$, denoted by $A$, which records all adjacent roads for each road segment $r \in \mathcal{R}$ by $A_{r}$ where

$$
A_{r}=\left\{r, r^{\prime} \in \mathcal{R} \mid r . s=r^{\prime} . \text { e or } r . e=r^{\prime} . s\right\}
$$


Then $A$ contains all $A_{r} \mid r \in \mathcal{R}$ and has the dimension of $|\mathcal{R}| \times$ $D$, where $D=\max \left\{\left|A_{r}\right|, \forall r \in \mathcal{R}\right\}$, i.e., the maximum number of adjacent roads for all roads in $\mathcal{R}$. For the road network represented in Figure 2, we have $|\mathcal{R}|=5$ and $D=3$ since we consider the directly connected roads to $r$ and also $r$ itself. Since the adjacent segment sets for different roads have different sizes, we pad each empty column in $A$ with a virtual road $r^{v}$ which has no adjacent roads and has a very small value of energy consumption so that when it is scaled, its value becomes -1 . During training and testing we are including the virtual road $r^{v}$ in the input training data, but on the output of training data, we remove it, so our network is not learning to predict the $r^{v}$ but it is acting as padding in our data-set.

Residual Unit: The components of the residual unit are given in the Figure 3. It contains three combinations of "Look Up layer $(\mathrm{LK})+$ Convolution (Conv) + Batch Normalization (BN)". A fusion of both energy consumption matrix $X_{r, t}$ and speed matrix $Z_{r, t}$ is required to obtain a single matrix for the energy consumption and speed for a pair of road segment and time-slot, denoted by $(X, Z)_{r, t}$. First, we feed it into the residual block. A look-up layer is required to embed the road network topology. A stack of convolutions is used in a single residual unit to understand connections between locations with a far distance. We attempt batch normalization after Conv layer for faster training speed.

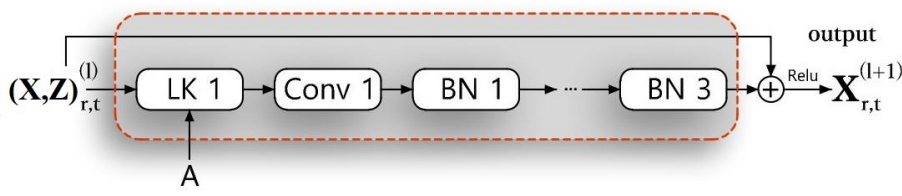

Figure 3: Residual Unit.

Look-up Operation: Given the energy consumption and traffic speed data recorded in $(X, Z)_{r, t}$, the topology of road network represented by the adjacent road matrix $A$ is embedded as follows:

$$
\mathcal{X}^{(l)}=L K\left((X, Z)_{r, t}^{(l)}, A\right)
$$

This operation aims to perform a look-up in the embedding matrix and returns the embedding of $A$. An embedding matrix would look like a vector of energy and speed values of the adjacent roads in $A$ from the matrix $(X, Z)_{r, t} . \mathcal{X}^{(l)}$ represents the result of the look-up operation and contains not only the energy and speed values of $|\mathcal{R}|$ roads over $n$ time-slots, but also the energy and speed values of their adjacent roads and has the dimension $|\mathcal{R}| \times D \times n$. Note that the virtual road added $r^{v}$ is acting as a placeholder in LK operation.

Figure 4 describes the LK result with more details. To model traffic conditions, LK constructs a tensor $\mathcal{X}^{(l)} \in \mathbb{R}^{|\mathcal{R}| \times D \times n}$, with the three dimensions standing for road segments, adjacent roads and time-slots, respectively. As shown in Figure 4 , an entry $\mathcal{X}^{(l)}(i, j, k)=$ $x$ denotes energy and speed values of the $j^{\text {th }}$ adjacent road of the road $i$ in time-slot $k$.

Convolution: The input of the convolution layer is traffic road tensor $\mathcal{X}^{(l)}$. We use a 3D convolution layer to extract the features of spatial matrix as shown in Figure 5. Output layer is to generate the energy prediction considering speed feature. At an arbitrary $l$-th layer, we use $k^{(l)}$ filters to convolve and concatenate all matrices

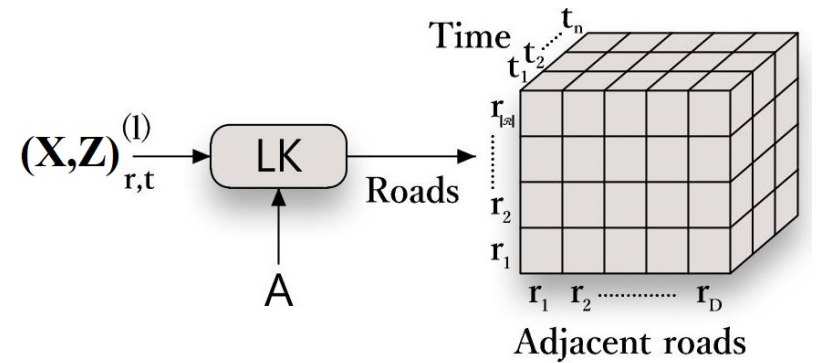

Figure 4: Look-Up Operation.

to get $\mathcal{X}^{(l+1)}$. The $k$-th matrix convolved by the $k$-th filter can be formulated as follows

$$
x^{l, k}=\left[x_{1}^{l, k}, \ldots, x_{i}^{l, k}, \ldots, x_{|\mathcal{R}|}^{l, k}\right]
$$

where

$$
x_{i}^{l, k}=f\left(L K\left((X, Z)_{r, t}^{(l-1)}, A\right) * W^{l, k}+b^{l, k}\right)
$$
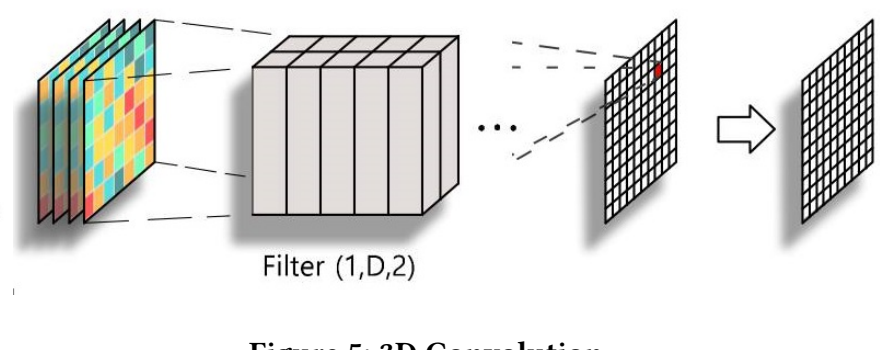

Filter $(1, D, 2)$

Figure 5: 3D Convolution.

Here, * denotes the convolution operation which uses the $k$ th filter $W^{l, k}, f$ is an activation function, e.g. the rectifier ReLU $f(x)=\max (0, x)$ which has achieved a training effectiveness in reducing the problem of gradient vanishing, $W^{l, k}$ and $b^{l, k}$ are the learnable parameters in the $l$-th layer with the $k$-th filter. We use a $3 \mathrm{D}$ filter of dimension $1 \times D \times 2$ where $D$ is the columns number of adjacent road matrix $A$. This filter aims at emphasizing the spatial features of adjacent roads.

To model large citywide dependencies and connect far roads, we still need deep networks to have more consecutive layers. To address this issue, we employ residual learning [11] in our model. We stack several residual units as follows:

$$
(X, Z)_{r, t}^{(l+1)}=(X, Z)_{r, t}^{(l)}+\mathcal{F}\left((X, Z)_{r, t}^{(l)}, \theta^{(l)}\right)
$$

where $(X, Z)_{r, t}^{(l)}$ and $(X, Z)_{r, t}^{(l+1)}$ are the input and output of the $l$-th residual unit, respectively; $\mathcal{F}$ is the residual function, e.g. a stack of three combinations of "LK $+\mathrm{Conv}+\mathrm{BN}$ " as shown in Figure 3 and $\theta^{(l)}$ includes all learnable parameters in the $l$-th residual unit.

\subsection{Temporal Features Extraction}

After $K$ residual units, we use the RNN model to learn the long-term temporal patterns considering the surrounding area. On top of the $K$ residual units, we get the output tensor $(X, Z)^{K} \in \mathbb{R}^{n \times|\mathcal{R}| \times f_{K}}$ where $f_{K}$ is the number of the convolution filters at the last $K$-th Conv layer. We reshape $(X, Z)^{K}$ in the way of time sequence to 
feed into RNN layer. We get a tensor $X^{\prime} \in \mathbb{R}^{|\mathcal{R}| \times n \times f_{K}}$ representing the speed and energy vectors for road $r_{i}$ in $n$ time-slots, where

$$
X_{i, t}^{\prime}=(X, Z)^{K}[t, i,:]
$$

As a variant of RNN, LSTM is good at learning long time-series patterns avoiding the problem of vanishing gradient [3]. Compared with LSTM, bidirectional LSTM (Bi-LSTM) [10] utilizes additional backward information and thus enhances the memory capability. We follow the same way to capture the temporal information of every time interval.

Because we need to extract patterns from speed, it is necessary to note that traffic speed is affected by daily, weekly, or occasional events. For instance, traffic patterns on Monday and Friday may be very different though both are workdays. Thus, to learn the long and short term energy consumption dynamics, we train the vector $X_{i, t}^{\prime}$ which records the energy and speed on $\operatorname{road} r_{i} \in \mathcal{R}$ for $n$ time-slots.

The structure of a bidirectional LSTM can be represented by two types of connections, one going forward in time, which helps us learn from previous representations and another going backwards in time, which helps us learn from future representations.

When feeding $X_{i, t}^{\prime}$ into Bi-LSTM, we get the $i$-th hidden states $\vec{h}_{i}$ and $\overleftarrow{h}_{i}$ of the forward and backward layers, respectively. We then concatenate these two states to get the $i$-th hidden state $h_{i}=$ $\left[\vec{h}_{i}, \overleftarrow{h}_{i}\right]$

For a given LSTM cell, the input gate, the forget gate, the output gate and the input cell state can be calculated using the following equations:

$$
\begin{aligned}
i_{t} & =\sigma_{g}\left(w_{i} X_{i, t}^{\prime}+u_{i} h_{t-1}+b_{i}\right) \\
f_{t} & =\sigma_{g}\left(w_{f} X_{i, t}^{\prime}+u_{f} h_{t-1}+b_{f}\right) \\
o_{t} & =\sigma_{g}\left(w_{o} X_{i, t}^{\prime}+u_{o} h_{t-1}+b_{o}\right) \\
g_{t} & =\tanh \left(w_{g} X_{i, t}^{\prime}+u_{g} h_{t-1}+b_{g}\right)
\end{aligned}
$$

where $w_{i}, w_{f}, w_{o}$ and $w_{g}$ the weight matrices mapping the hidden layer input to the three gates and the input cell state, while $u_{i}, u_{f}, u_{o}$ and $u_{g}$ are the weight matrices connecting the previous cell output state to the three gates and the input cell state. $b_{i}, b_{f}, b_{o}$ and $b_{g}$ are four bias vectors. The $\sigma_{g}$ is the gate activation function, e.g. the Sigmoid function $\sigma(a)=1 /\left(1+e^{-a}\right)$, and the tanh is the hyperbolic tangent function. Then the memory cell and hidden state are updated as:

$$
\begin{aligned}
c_{t} & =f_{t} * c_{t-1}+i_{t} * g_{t} \\
h_{t} & =o_{t} * \tanh \left(c_{t}\right)
\end{aligned}
$$

The forward layer output sequence, $\vec{h}_{i}$, is iteratively calculated using inputs in a positive sequence from time $t-n$ to time $t$, while the backward layer output sequence, $\overleftarrow{h}_{i}$, is calculated using the reversed inputs from time $t-n$ to time $t$. The Bi-LSTM layer generates an output vector, $E_{T}$ in which each element is calculated by using the following equation:

$$
E_{i}=\sigma\left(\vec{h}_{i}, \overleftarrow{h}_{i}\right)
$$

where $\sigma$ function is used to combine the two output sequences. Similar to the LSTM layer, the final output of a Bi-LSTM layer can be represented by the Spatio-Temporal vector $E_{S T}$ in which the last element is the predicted energy consumption for the next time iteration such as

$$
E_{S T}=\left[E_{i, t-n}, \ldots, E_{i, t}, E_{i, t+1}\right]
$$

\subsection{Weather and Periodicity Features Extraction}

In addition to the vehicle, speed, spatial and temporal features, the energy consumption is affected by other complex factors such as periodicity. We extract and learn these information to have more accurate prediction. If we consider a single road segment, we can observe that the traffic dynamic changes periodically, thus, the energy consumption could be similar at the same time intervals on different days. We mainly consider three kinds of periodicity:

- Inter-time-intervals Periodicity: Traffic condition in a region is affected by recent time intervals. For instance, a traffic congestion occurring at $8 \mathrm{am}$ will affect that of $9 \mathrm{am}$. Same behaviour can be adopted by the energy consumption because of the high correlation between EC and traffic condition. We construct $P_{\text {int }}$ to design the dependency between timeintervals.

- Daily Periodicity: we can see the daily periodicity denoted by $P_{d}$, especially at weekdays, i.e., the traffic condition at a given time interval (17:00pm-17:15pm) of Wednesday can be similar to the next following day, in the absence of special condition.

- Weekly Periodicity: at a given time interval (8:00pm-8:30pm) of Monday, the traffic condition shows a similar trend at the same time interval of the previous Monday on previous weeks, which is the weekly periodicity denoted by $P_{w}$.

Using a fully connected layer, we train $P_{i n t}, P_{w}$ and $P_{d}$ to get $E_{i n t}, E_{w}$ and $E_{d}$, respectively. The predicted energy consumption value respecting the periodicity factor $E_{P}$ is obtained such as $E_{P}=$ $E_{w} \oplus E_{d} \oplus E_{\text {int }}$ where $\oplus$ is the concatenation of the results to get $E_{P}$.

In addition, observations on traffic dynamics have demonstrated that the traffic conditions is significantly influenced by weather [16]. This is because the traffic speed in the heavy rain will be slower than that in sunny days because of safe driving. Thus, the energy consumption should be affected. Weather data including temperature are extracted from public data-sets using a parser. We incorporate the attributes of the weather conditions (rainy/sunny/windy etc.). Note that these factors are categorical values which can not feed to the neural network directly. In our model, we use one hot encoding method to convert categorical data to a low-dimensional integer vector. Formally, a fully connected layer is adopted to extract weather information and get $E_{W}$.

Then, as shown in Figure 1, sharing the same shape, $E_{P}, E_{W}$ and $E_{S T}$ are combined.

\subsection{Fusion}

Predicting the energy consumption by using only vehicle features is not enough to have an accurate prediction. The spatio-temporal learning, added to vehicle and speed pattern extraction significantly helps to get more accurate energy prediction. For this reason, we merge the output of all the components, i.e., stacked residual neural 
networks and bidirectional LSTM, with that of the periodicity and weather components as shown in Figure 1. Finally, the predicted value at the $t$-th time interval, denoted by $\widetilde{E}_{t}$, is defined as

$$
\widetilde{E}_{t}=\tanh \left(W_{S T} \circ E_{S T}+W_{P} \circ E_{P}+W_{W} \circ E_{W}\right)
$$

where $\circ$ is Hadamard product (i.e., element-wise multiplication), $W_{S T}, W_{W}$ and $W_{P}$ are the learnable parameters.

To predict $E_{t}$, our model can be trained by minimizing mean squared error $\mathcal{L}(\theta)$ between the predicted energy matrix and the true energy vectors such as

$$
\mathcal{L}(\theta)=\left\|E_{t}-\widetilde{E}_{t}\right\|_{2}^{2}
$$

where $\theta$ are all learnable parameters in DeepFEC model.

\section{EXPERIMENTS}

\subsection{Data-set Description}

In our experiment, we used two data-sets to evaluate our model. Details are given as follows:

- The Safety Pilot Model Deployment (SPMD) ${ }^{1}$ database contains driving records of roughly 3,000 drivers in Ann Arbor, MI. Data were extracted between 10-05-2015 and 10-18-2015 by the University of Michigan Transportation Research Institute.

- The Vehicle Energy Data-set (VED) ${ }^{2}$ is a public data-set providing the rate of fuel and energy consumption timeseries data of various personal cars operated in diverse realworld driving conditions in Michigan, USA, over a year from November 2017 to November 2018 . The data-set contains data collected from 366 vehicles. The diverse fleet includes passenger cars (coupes, hatchbacks, sedans, convertibles, crossovers, and luxury cars) and light trucks (pickup trucks, SUVs, minivans, and wagons).

VED includes a total of 357,000 miles of naturalistic driving data from the fleet. The data-set consists of two primary components: static data and dynamic time-series data.

5.1.1 static data. include vehicle parameters such as vehicle type, engine configuration, engine displacement and vehicle weight. Different parameter values are detailed in Table 2.

Table 2: Vehicle's Parameters

\begin{tabular}{cc}
\hline Parameter & Values \\
\hline Vehicle Type & ICE Vehicle, HEV, PHEV, EV \\
\hline Engine Configuration & I4, V4, V4 Flex, V6 PZEV \\
\hline Engine Displacement & $1.0 \mathrm{~L}, 2.0 \mathrm{~L}, 3.6 \mathrm{~L}$ \\
\hline Vehicle Weight & $2500 \mathrm{~kg} 6000 \mathrm{~kg}$ \\
\hline
\end{tabular}

Table 3 provides details on the number of vehicles and total mileages each group accumulated. Among total 357,000 miles traveled by the fleet, $55.7 \%$ of them are from ICE vehicles.

\footnotetext{
${ }^{1}$ https://catalog.data.gov/dataset/safety-pilot-model-deployment-data

${ }^{2}$ https://github.com/gsoh/VED
}

Table 3: Number of Vehicles and Mileage of the Fleet

\begin{tabular}{ccc}
\hline Vehicle Type & \# of Vehicles & Mileage \\
\hline ICE & 249 & 199,000 \\
\hline HEV & 90 & 109,000 \\
\hline PHEV & 24 & 45,000 \\
\hline EV & 3 & 4,000 \\
\hline Total & 366 & $\mathbf{3 5 7 , 0 0 0}$ \\
\hline
\end{tabular}

5.1.2 Dynamic time-series data. The data include vehicle speed, vehicle energy consumption, engine related signals and ambient air temperature. The timestamps, GPS points (latitude and longitude) are also reported.

\subsection{Pre-Processing}

In addition to energy consumption data, we have extracted the road network of Michigan state, which is mainly a geographic data of the area.

We represent the road network as a directed graph composed of road segments and nodes, each node is an intersection of two road segments. Each directed link is a road segment connecting two nodes. A road consists of multiple links, two-way streets are often represented as two directed links with opposite directions. The longitude and latitude of each node are recorded. We calculate the average energy consumption for each link at a particular hour. To learn the short-term traffic periodicity, we divide a day $\delta$ into different time-bins (e.g. an hour is a bin in our experiments).

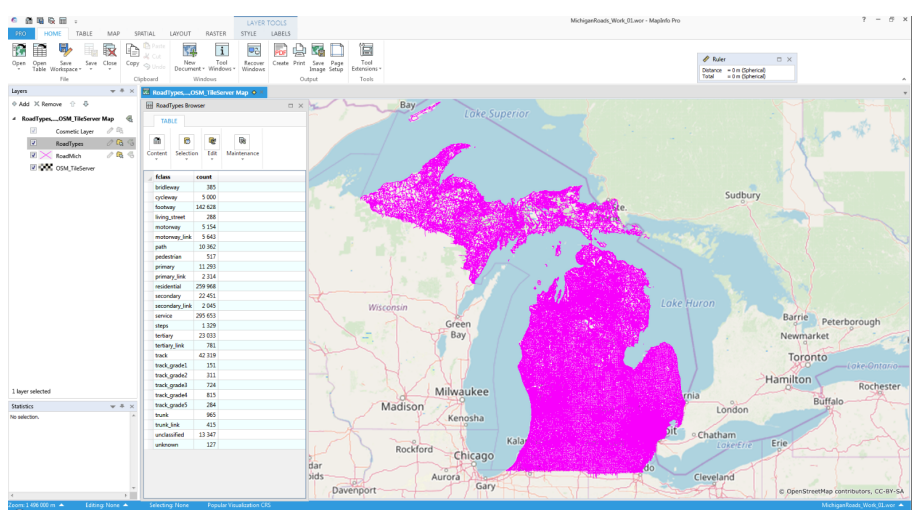

Figure 6: Michigan Road Network Extraction.

Figure 6 illustrates a geographic heat-map for the traces of the extracted road network. As shown in Figure 7, a link represents the road segment that connects two consecutive intersections. Each energy consumption value depicted in the dynamic time-series data is matched to a link from Michigan road network by using the geographic coordinates of both links and GPS points related to energy consumption data. Since some historical energy consumption data are not available in some roads, we use energy consumption value at nearby roads in the same time-slot or EC value at closest time-slot for the same road.

To scale the data into the range $[-1,1]$, we use the Min-Max normalization method and then, for comparison with real energy 


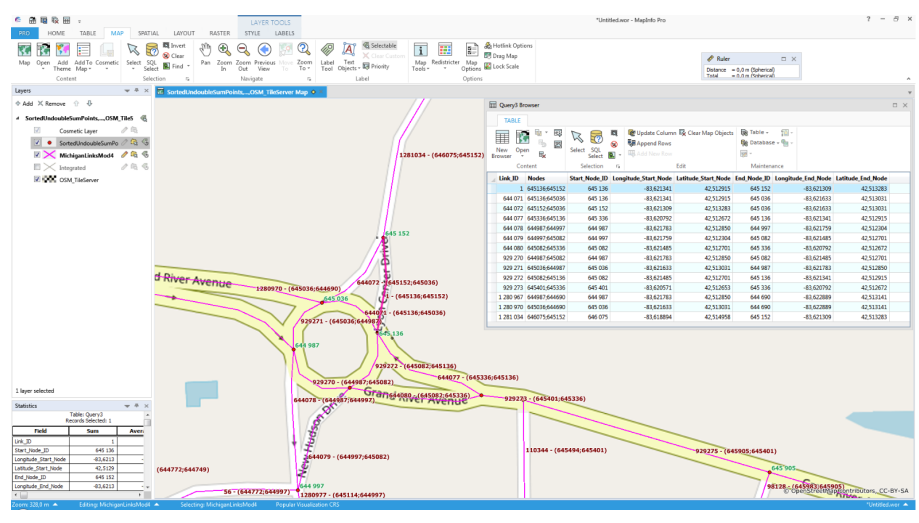

Figure 7: Michigan link’s Extraction.

consumption data, we re-scale the predicted value back to the normal values. For vehicle, weather and periodicity features, a binary vector is given by one-hot coding to transform static vehicle data, weather data and the time-stamp of the day.

\subsection{Benchmarks}

Several prevailing algorithms are chosen for comparisons with our proposed model DeepFEC.

- Multivariate Regression (MR) [4]: used for estimating the link energy consumption where independent variables included link average traffic speed and road grade.

- SVR [27]: used for travel time estimation, Support Vector Regression (SVR) is a powerful regression method.

- ANNs: used for energy consumption prediction in $[7,19]$

- RNN [28]: a recurrent neural network (RNN), used for predicting temporal sequence values.

- GRU [8]: Gated Recurrent Unit (GRU) is an improved version of standard recurrent neural network to keep information from long ago, without washing it through time or remove information which is irrelevant to the prediction.

- LSTM [18]: Long short-term memory (LSTM) were developed to deal with the vanishing gradient problems that can be encountered when training traditional RNNs.

- Bi-LSTM [6]: Using bidirectional LSTM will run the inputs in two ways, one from past to future and one from future to past to measure the backward dependency of traffic data prediction.

- DCNN [17]: used to predict traffic speed, it consists of convolution, pooling and fully connected layers.

- ST-ResNet [31]: used for traffic prediction by employing residual network to model temporal dependencies .

- LC-RNN [16]: used for speed prediction, it consists of convolution, $\mathrm{RNN}$ and fully connected layers.

DCNN, ST-ResNet and LC-RNN aim to predict speed and traffic over a road network. Their input is a spatial speed matrix, i.e., speed for each road segment in $\mathcal{R}$. To evaluate these methods on EC prediction, we use the energy consumption tensor as an input.

\subsection{Performance}

We train our network with the following hyper-parameters setting: mini-batch size (48), learning rate (0.001) with adam optimizer, 1 $\times D$ filters and $2 \times D$ filters in each Convolution layer where $D$ is the maximum number of adjacent roads as described in Section 4.3. Afterwards, we continue to train the model on the full training data for a fixed number of epochs (e.g., 100, 500, 1000 epochs). The last week ( 7 days) is chosen as the validation set with 3 early stopping.

To learn the periodicity dynamics, we empirically fix it to oneday and one-week.

5.4.1 Evaluation Metrics. We measure our method by Root Mean Square Error (RMSE), Mean Absolute Error (MAE) and Mean Absolute Percentage Error (MAPE) where:

$$
\begin{gathered}
R M S E=\sqrt{\frac{1}{n} \sum_{t=1}^{n}\left(E_{t}-\hat{E}_{t}\right)^{2}} \\
M A E=\frac{1}{n} \sum_{t=1}^{n}\left|E_{t}-\hat{E}_{t}\right| \\
M A P E=\frac{100 \%}{n} \sum_{t=1}^{n}\left|\frac{E_{t}-\hat{E}_{t}}{E_{t}}\right|
\end{gathered}
$$

with $\hat{E}_{t}$ and $E_{t}$ are the predicted energy consumption value and real energy consumption value, respectively; $n$ is the number of all predicted values.

5.4.2 Results on VED Data-sets. Table 4 shows the RMSE, MAE and MAPE of the most existing models dealing with different traffic prediction problems. We compare our model DeepFEC with the 10 other benchmarks using VED data-set. The time interval is one

\begin{tabular}{c|c|c|c|c} 
Methods & RMSE & MAE & MAPE & Time $(\mathrm{min})$ \\
\hline MR & 2.475 & 1.512 & 0.151 & 0.63 \\
SVR & 2.512 & 1.534 & 0.153 & 0.82 \\
ANNs & 0.833 & 0.510 & 0.051 & 0.94 \\
LSTM & 0.739 & 0.451 & 0.045 & 2.6 \\
GRU & 1.994 & 1.218 & 0.121 & 1.5 \\
Bi-LSTM & 0.778 & 0.475 & 0.047 & 1.43 \\
RNN & 0.819 & 0.500 & 0.050 & 0.94 \\
DCNN & 1.909 & 1.166 & 0.116 & 0.41 \\
LC-RNN & 0.993 & 0.606 & 0.060 & 1.68 \\
ST-ResNEt & 1.067 & 0.651 & 0.065 & 2.22 \\
\hline DeepFEC $[\mathbf{o u r s}]$ & $\mathbf{0 . 4 7 4}$ & $\mathbf{0 . 2 9 0}$ & $\mathbf{0 . 0 2 9}$ & $\mathbf{1 . 3 5}$ \\
DeepFEC $\backslash P r$ & 0.630 & 0.384 & 0.038 & 1.27 \\
DeepFEC $\backslash W$ & 0.475 & 0.291 & 0.029 & 1.34 \\
DeepFEC $\backslash S p$ & 0.694 & 0.424 & 0.042 & 1.14
\end{tabular}

Table 4: Results on VED Data-set.

hour and the default number of intervals to be predicted is 1, i.e., we predict energy consumption in the next hour of each day in the validation set. Meanwhile, we compare all 3 variants of DeepFEC with different components: DeepFEC is our model as described in the Figure 1, in DeepFEC $\backslash \operatorname{Pr}$ and DeepFEC $\backslash W$ models, we remove the periodicity and the weather learning, respectively, to measure their effects and in DeepFEC $\backslash S p$, we evaluate the impact of capturing the speed patterns. As shown in Table 4, a very slight improvement 
in terms of accuracy is reported when discarding the weather component. That is because the training and testing data are from same season. It may have significant improvement if collected data are from different seasons. In addition, as detailed in Figure 9a, discarding the periodicity learning may reduce the accuracy achieved by DeepFEC but not as much as the speed learning does, which confirms the strong correlation between energy consumption and speed. Thus, considering the speed dimension in predicting the energy consumption significantly improves accuracy. From the same table, we observe that all these three models are better than the 10 benchmarks. And we can find that DeepFEC reduces error to 0.474 .

Figure 8a shows the fuel consumption rates $(\mathrm{CR})$ as well as the estimated results reported in different road segments. We present the evaluation of our approach at a morning period (11AM). We also show the accuracy of our method comparing the real consumption rate and the predicted consumption rate for different road sections: (i) Link 1: situated in a parking place at New Hudson, MI 48165, (ii) Link 2: is the Hickory Pointe Boulevard, Ypsilanti, MI 48197 at an urban area and (iii) Link 3: represents a section from Farmington Hills highway. The results show detailed examples of how our model preforms while changing real-life traffic conditions. For instance, a good accuracy is shown for link 1 (real CR 0.874 vs predicted CR 0.715$)$ when speed is low $(25.65 \mathrm{~km} / \mathrm{h})$ in a parking place, for link 2 (1.372 vs 1.405$)$ when speed is high $(78 \mathrm{~km} / \mathrm{h})$ as well as for link 3 (2.021 vs 1.967$)$ with higher speed $(110 \mathrm{~km} / \mathrm{h})$. Figure $8 \mathrm{~b}$ presents the predicted fuel consumption rates (CR) for link 2 at morning period (11AM) given by the benchmark models as well as our proposed model.

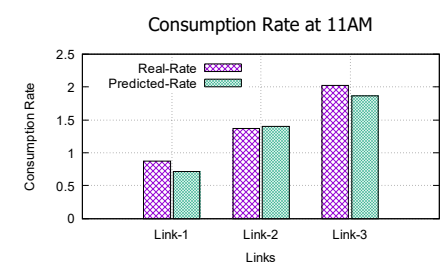

(a) [Accuracy at Morning Period

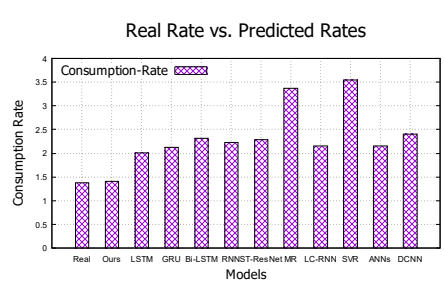

(b) Different Models Performances
Figure 8: Real consumption rate vs, Predicted consumption rate.

In addition, Table 4 shows a study of the computational efficiency. The results show that MR, SVR, ANNs, RNN and DCNN have better computational performances, however, in terms of accuracy, our model by far outperforms these approaches. In addition, our approach has better time cost than LSTM, GRU, Bi-LSTM, LC-RNN and ST-ResNet. That is because the computational cost of deep learning models essentially depends on the number of epochs used to achieve the best accuracy. The key for our computational efficiency is that our model gives its best accuracy after 40 epochs, while other testing models may require more epochs. A good trade-off between accuracy and computational cost is offered by our model. Please note that the reported results are for energy prediction for a large road network. However, in real-time applications, our method will handle a sub-graph of the road network, just roads around the vehicle location, then just a few seconds will be required for training the model.

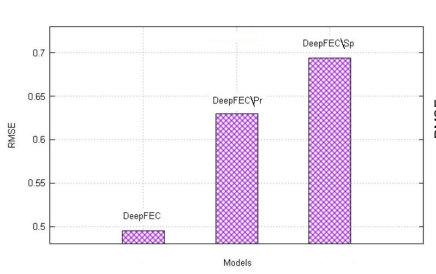

(a) Speed and Periodicity Effect

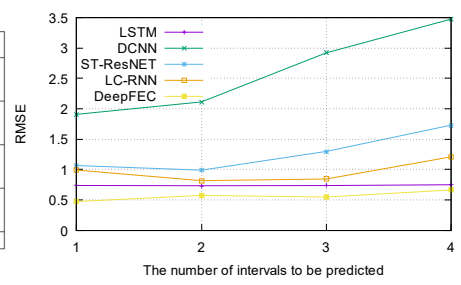

(b) Predicted time-intervals Effect
Figure 9: Parameters Effect.

The prediction of energy consumption rate is impacted by several factors. We mainly study four kinds of influence:

Influence of driving environments on EC prediction: In Michigan road network, three driving environments (urban roads, highways and downtown) are present in our data-set. We show how the EC prediction is affected by each of them.

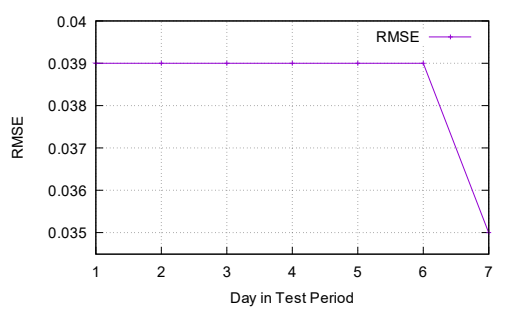

(a) Prediction on Urban Road

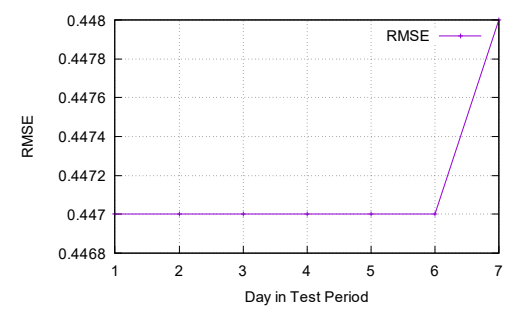

(b) Prediction on Highway

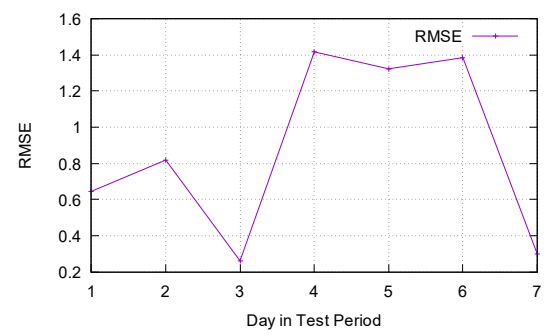

(c) Prediction on Downtown

Figure 10: Prediction on different types of Roads.

- Urban Road: we observe the prediction behaviour of our model in a section of Plymouth road in North Ann Arbor, Michigan, which has two lanes in each direction and two consecutive signalized intersections, Plymouth Nixon and Plymouth-Huron Parkway. It represents the busy urban roads with closely-spaced traffic lights.

- Highway: the second observation is in a section of M5 Farmington Hills, Michigan, which consists of one direction lane. This section represents the highway driving environment. 
- Downtown: we choose a double-lane road in downtown Ann Arbor, Geddes Avenue in Central Campus Medical as an observation area. This environment includes two of the busiest signalized intersections in the city of Ann Arbor. This section represents a districts with heavy vehicle and pedestrian traffic.

As shown in Figure 10, prediction in urban road with error of 0.039 is better than prediction in both highway and downtown with errors greater than 0.3 . In addition, comparing to prediction in downtown area, prediction in both urban environment and highway shows a constant behaviour during the first 6 days and slightly changes in the last day. However in downtown area, there is no stability in the prediction behaviour and the error is high and achieves RMSE of 1.41. That is because this driving environment is always cloudy with heavy vehicle and pedestrian traffic. Thus the traffic patterns are not well captured.

Influence of predicted time-interval on EC prediction:

The default parameter of predicted time-intervals, denoted $p$, is 1 hour. Figure 9b shows the comparative performances for LSTM, DCNN, ST-ResNet, LC-RNN and DeepFEC varying the number of predicted intervals from 1 to 4 . LSTM shows a better performance against DCNN and ST-ResNET since it is good in capturing timeseries patterns. However, DCNN and ST-ResNET are not. Since LC-RNN is an hybrid model capturing both spatial and temporal features, it shows better results than DCNN. Note that the performance becomes worse with larger $p$ since the correlation between value in current moment and predicted value in next time-intervals decreases. Slight decrease of DeepFEC error when varying $p$ which shows its capacity of dealing with long-term prediction. Especially, when DeepFEC still has the best performance comparing to other models.

Influence of vehicle type on EC prediction: Four vehicle types (ICE, HEV, PHEV and EV) are present in our data-set to provide EC rate in different locations in Michigan road network. It is important to analyse how our model deals with each type of vehicle.

In the following, we give the parameters of the observed vehicles as well as its driving environment. Since the prediction in urban area and highway is stable and offers lower errors as described above, we observe vehicles on these driving environments. This allows us to analyse the real effect of vehicle type on prediction.

- ICE Vehicle: 4-FI 1.5 (transmission: 5-SP AUTOMATIC), the weight is $2500 \mathrm{~kg}$. observed in the highway Farmington Hills

- HE Vehicle: the car characteristics are: 4-GAS/ELECTRIC $1.8 \mathrm{~L}$, the weight is $3000 \mathrm{~kg}$, observed in the urban way, Golden Valley Drive, New Hudson.

- PHE Vehicle: the car characteristics are: 4-GAS/ELECTRIC $2.0 \mathrm{~L}$, weight is $4000 \mathrm{~kg}$, observed in the urban way, 7154 Wilson Street, Dexter.

- Electric Vehicle: the car characteristics are: ELECTRIC, weight is $3500 \mathrm{~kg}$, observed in South Maple, Ann Arbor which is considered as urban road.

In Figure 11, we study the behaviour of our prediction model on different vehicle types during the 7 days of validation set.

Comparing to the global error (RMSE) given by DeepFEC model in Table 4, i.e., 0.474, ICE vehicles show a similar error, i.e., 0.447 .
That is because in our data-set, more than 240 vehicles (for a total of 366 vehicles, as shown in Table 3) providing energy consumption rate are ICE vehicles. However, HEV and PHEV have errors between 0.1 and 0.2 which are lower than the global model error, which shows DeepFEC capacity of capturing new vehicles patterns. The study on electric vehicles shows that almost predicted values have errors $\geq$ than the global error. Taking into consideration the relative rarity of these vehicles (only 3 electric vehicles in our data-set), the behaviour of our prediction model is justified.

In summary, from the above experimental results, we can observe that DeepFEC achieves the best performance compared with the state-of-the-arts. Moreover, the fusion of spatial dependency on the road network, temporal evolution and contextual information are the key factors to learn more from the energy consumption behaviour.

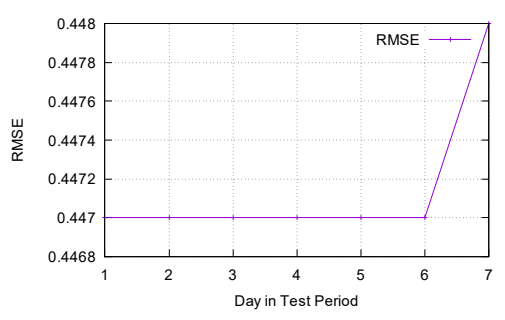

(a) Prediction for ICE Vehicle

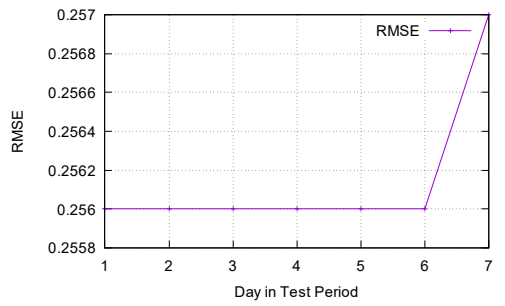

(c) Prediction for PHEV

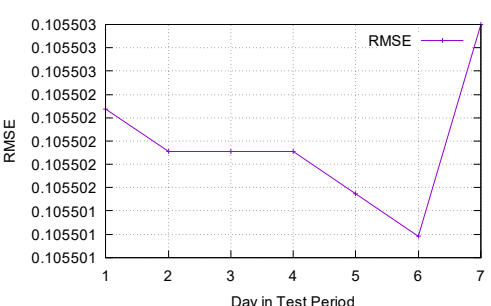

(b) Prediction for HEV

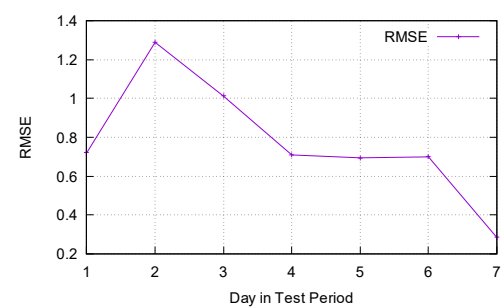

(d) Prediction for EV
Figure 11: Prediction for different Vehicle Types.

5.4.3 Results on SPMD Data-sets. We evaluate our model DeepFEC using SPMD data-sets. Table 5 shows the RMSE and MAE of several related works as well as DeepFEC model. Evaluation shows similar results as VED data-sets.

\begin{tabular}{c|c|c|c} 
Methods & RMSE & MAE & Time (min) \\
\hline MR & 2.725 & 2.475 & 0.57 \\
ANNs & 0.913 & 0.675 & 0.82 \\
LSTM & 0.793 & 0.577 & 2.40 \\
Bi-LSTM & 0.812 & 0.628 & 1.23 \\
RNN & 0.918 & 0.728 & 0.56 \\
DCNN & 1.998 & 1.291 & 0.37 \\
ST-ResNEt & 1.125 & 1.019 & 2.22 \\
\hline DeepFEC [ours] & $\mathbf{0 . 5 0 8}$ & $\mathbf{0 . 3 1 2}$ & $\mathbf{1 . 0 1 1}$
\end{tabular}

Table 5: Results on SPDM Data-sets. 
The results reported in the paper are conducted in a large road network to show that our model performs well in a such conditions which is very benefit for the real-world applications in transportation network companies such as taxi or bus markets that can hold a large vehicle fleet and want to predict the consumption rates on a large road network. Add to that, our proposed model enables the users to select a region (a sub-graph from the road network) or some given links to predict their energy consumption rates which is useful for real-time applications.

\section{CONCLUSION}

This paper proposes a novel deep learning based energy consumption prediction method that can extract spatio-temporal traffic features in addition to contextual patterns. We evaluate our model on Michigan road network, achieving performances which are significantly beyond ten existing methods, confirming that our model is applicable to the energy consumption prediction problem. An interesting future direction is to analyse the effect of traffic lights to better capture traffic patterns in downtown areas.

\section{REFERENCES}

[1] Matthew Barth and Kanok Boriboonsomsin. 2008. Real-World CO2 Impacts of Traffic Congestion. University of California Transportation Center, Working Papers (01 2008).

[2] Matthew Barth, George Scora, and Theodore Younglove. 2004. Modal Emissions Model for Heavy-Duty Diesel Vehicles. Transportation Research Record 1880, 1 (2004), 10-20.

[3] Y. Bengio, P. Simard, and P. Frasconi. 1994. Learning long-term dependencies with gradient descent is difficult. IEEE Trans. Neural Networks 5, 2 (1994), 157-166.

[4] K. Boriboonsomsin, M. J. Barth, W. Zhu, and A. Vu. 2012. Eco-Routing Navigation System Based on Multisource Historical and Real-Time Traffic Information. IEEE Trans. on Intell. Trans. Sys 13, 4 (2012), 1694-1704.

[5] Shiyu Chang, Wei Han, Jiliang Tang, Guo-Jun Qi, Charu C. Aggarwal, and Thomas S. Huang. 2015. Heterogeneous Network Embedding via Deep Architectures. In The 21th ACM SIGKDD (KDD). 119-128.

[6] Zhiyong Cui, Ruimin Ke, and Yinhai Wang. 2018. Deep Bidirectional and Unidirectional LSTM Recurrent Neural Network for Network-wide Traffic Speed Prediction. CoRR abs/1801.02143 (2018).

[7] Yiman Du, Jianping Wu, Senyan Yang, and Liutong Zhou. 2017. Predicting vehicle fuel consumption patterns using floating vehicle data. Environmental Sciences 59 (2017), $24-29$.

[8] R. Fu, Z. Zhang, and L. Li. 2016. Using LSTM and GRU neural network methods for traffic flow prediction.. In The 31st YAC. 324-328.

[9] Qiang Gao, Fan Zhou, Kunpeng Zhang, Goce Trajcevski, Xucheng Luo, and Fengli Zhang. 2017. Identifying Human Mobility via Trajectory Embeddings. In The 26th IFCAI. 1689-1695.

[10] A. Graves and J. Schmidhuber. 2005. Framewise phoneme classification with bidirectional LSTM networks. In IEEE Int. Joint Conf. on Neural Networks., Vol. 4. 2047-2052 vol. 4.

[11] Kaiming He, Xiangyu Zhang, Shaoqing Ren, and Jian Sun. 2015. Deep Residual Learning for Image Recognition. CoRR abs/1512.03385 (2015).

[12] Miyeon Jeon, Yoojeong Noh, Yongwoo Shin, O-Kaung Lim, Inwon Lee, and Daeseung Cho. 2018. Prediction of ship fuel consumption by using an artificial neural network. Mechanical Science and Technology 32, 12 (2018), 5785-5796.

[13] Alex Krizhevsky, Ilya Sutskever, and Geoffrey E. Hinton. 2012. ImageNet Clas sification with Deep Convolutional Neural Networks. In The 25th Int. Conf. on Neural Information Processing Systems - Volume 1 (NIPS). 1097-1105.

[14] Guoqiang Li and Daniel Görges. 2019. Ecological Adaptive Cruise Control and Energy Management Strategy for Hybrid Electric Vehicles Based on Heuristic Dynamic Programming. IEEE Trans. Intell. Trans. Sys. 20, 9 (2019), 3526-3535.

[15] Yaguang Li, Kun Fu, Zheng Wang, Cyrus Shahabi, Jieping Ye, and Yan Liu. 2018. Multi-task Representation Learning for Travel Time Estimation. In The 24th ACM SIGKDD (KDD). ACM, 1695-1704.

[16] Zhongjian Lv, Jiajie Xu, Kai Zheng, Hongzhi Yin, Pengpeng Zhao, and Xiaofang Zhou. 2018. LC-RNN: A Deep Learning Model for Traffic Speed Prediction. In The 27th IFCAI. 3470-3476.

[17] Xiaolei Ma, Zhuang Dai, Zhengbing He, and Yunpeng Wang. 2017. Learning Traffic as Images: A Deep Convolution Neural Network for Large-scale Transportation Network Speed Prediction. CoRR abs/1701.04245 (2017).
[18] Xiaolei Ma, Zhimin Tao, Yinhai Wang, Haiyang Yu, and Yunpeng Wang. 2015. Long short-term memory neural network for traffic speed prediction using remote microwave sensor data. Trans. Research Part C: Emerging Technologies 54 (2015), $187-197$.

[19] L. P. Perera and B. Mo. 2017. Machine Intelligence Based Data Handling Framework for Ship Energy Efficiency. IEEE Trans. on Vehicular Technology 66, 10 (2017), 8659-8666.

[20] David Schrank, Bill Eisele, and Tim Lomax. 2019. Urban Mobility Report. The Texas A\&M Transportation Institute (2019).

[21] R. Shankar and J. Marco. 2013. Method for estimating the energy consumption of electric vehicles and plug-in hybrid electric vehicles under real-world driving conditions. IET Int. Trans. Sys. 7, 1 (2013), 138-150.

[22] Toshiaki Uemura. 2019. Pre-Estimation of Electric Vehicle Energy Consumption on Unfamiliar Roads and Actual Driving Experiments. In VLDB 2019 PhD Workshop.

[23] Toshiaki Uemura, Yuta Kashiwabara, Daiki Kawanuma, and Takashi Tomii. 2016. Accuracy Evaluation by GPS Data Correction for the EV Energy Consumption Database. In The 13th Int. Conf. on Mobile and Ubiquitous Systems (Hiroshima, Japan) (MOBIQUTTOUS). ACM, 213-218.

[24] Jinghui Wang and Hesham Rakha. 2016. Fuel consumption model for conventional diesel buses. Applied Energy 170 (2016), $394-402$.

[25] Jinghui Wang and Hesham Rakha. 2017. Convex Fuel Consumption Model for Diesel and Hybrid Buses. 7. Transportation Research Board 2647 (01 2017), 50-60.

[26] Zheng Wang, Kun Fu, and Jieping Ye. 2018. Learning to Estimate the Travel Time. In The 24th ACM SIGKDD (KDD). ACM, 858-866.

[27] Chun-Hsin Wu, Jan-Ming Ho, and Lee D.T. 2004. Travel-time prediction with support vector regression. IEEE Trans. Intel. Trans. Sys 5, 4 (2004), 276-281.

[28] Hao Wu, Ziyang Chen, Weiwei Sun, Baihua Zheng, and Wei Wang. 2017. Modeling Trajectories with Recurrent Neural Networks. In The 26th IFCAI. 3083-3090.

[29] Tao Xu, Xiang Li, and Christophe Claramunt. 2018. Trip-oriented travel time prediction (TOTTP) with historical vehicle trajectories. Frontiers of Earth Science 12, 2 (Jun 2018), 253-263.

[30] Hanyuan Zhang, Hao Wu, Weiwei Sun, and Baihua Zheng. 2018. DeepTravel: a Neural Network Based Travel Time Estimation Model with Auxiliary Supervision. In $I F C A I$.

[31] Junbo Zhang, Yu Zheng, and Dekang Qi. 2017. Deep Spatio-Temporal Residual Networks for Citywide Crowd Flows Prediction. In $A A A I$.

[32] Junbo Zhang, Yu Zheng, Dekang Qi, Ruiyuan Li, and Xiuwen Yi. 2016. DNNbased Prediction Model for Spatio-temporal Data. In The 24th ACM SIGSPATIAL (GIS). ACM, 92:1-92:4.

[33] Qi Zhao, Qi Chen, and Li Wang. 2019. Real-Time Prediction of Fuel Consumption Based on Digital Map API. Applied Sciences 9, 7 (2019).

[34] Suping Zhao and Ye Yu. 2017. Effect of short-term regional traffic restriction on urban submicron particulate pollution. Environmental Sciences 55 (2017), 86 - 99. 